\title{
ARTICLE OPEN \\ High-fidelity resonant gating of a silicon-based quantum dot hybrid qubit
}

\author{
Dohun Kim ${ }^{1,2}$, Daniel R Ward ${ }^{1}$, Christie B Simmons ${ }^{1}$, Don E Savage ${ }^{3}$, Max G Lagally ${ }^{3}$, Mark Friesen ${ }^{1}$, \\ Susan N Coppersmith ${ }^{1}$ and Mark A Eriksson ${ }^{1}$
}

We implement resonant single qubit operations on a semiconductor hybrid qubit hosted in a three-electron Si/SiGe double quantum dot structure. By resonantly modulating the double dot energy detuning and employing electron tunnelling-based readout, we achieve fast ( $>100 \mathrm{MHz}$ ) Rabi oscillations and purely electrical manipulations of the three-electron spin states. We demonstrate universal single qubit gates using a Ramsey pulse sequence as well as microwave phase control, the latter of which shows control of an arbitrary rotation axis on the $X-Y$ plane of the Bloch sphere. Quantum process tomography yields $\pi$ rotation gate fidelities higher than $93(96) \%$ around the $X(Z)$ axis of the Bloch sphere. We further show that the implementation of dynamic decoupling sequences on the hybrid qubit enables coherence times longer than $150 \mathrm{~ns}$.

npj Quantum Information (2015) 1, 15004; doi:10.1038/npjqi.2015.4; published online 27 October 2015

\section{INTRODUCTION}

Isolated spins in semiconductors provide a promising platform to explore quantum mechanical coherence and develop engineered quantum systems. ${ }^{1-13}$ Silicon has attracted great interest as a host material for developing spin qubits because of its weak spin-orbit coupling and hyperfine interaction, and several architectures based on gate defined quantum dots have been proposed and demonstrated experimentally. ${ }^{14,15}$ Recently, a quantum dot hybrid qubit formed by three electrons in a double quantum dot was proposed, $^{16,17}$ and non-adiabatic pulsed-gate operation was implemented experimentally, ${ }^{18}$ demonstrating simple and fast electrical manipulations of spin states with a promising ratio of coherence time to manipulation time. However, the overall gate fidelity of the pulse-gated hybrid qubit is limited by relatively fast dephasing due to charge noise during one of the two required gate operations. Here we perform the first microwave-driven gate operations of a quantum dot hybrid qubit, avoiding entirely the regime in which it is most sensitive to charge noise. Resonant detuning modulation along with phase control of the microwaves enables a $\pi$ rotation time of $<5 \mathrm{~ns}(50 \mathrm{ps})$ around $X(Z)$ axis with high fidelities $>93(96) \%$. We also implement Hahn echo ${ }^{19-21}$ and Carr-Purcell $(C P)^{22}$ dynamic decoupling sequences with which we demonstrate a coherence time of over $150 \mathrm{~ns}$. We further discuss a pathway to improve gate fidelity to above $99 \%$, exceeding the threshold for surface code based quantum error correction. ${ }^{23}$

The quantum dot hybrid qubit combines desirable features of charge (fast manipulation) and spin (long coherence time) qubits. The qubit states can be written as $|0\rangle=|\downarrow\rangle|S\rangle$, where $S$ denotes a singlet state in the right dot, and $|1\rangle=1 / \sqrt{3}|\downarrow\rangle\left|T_{0}\right\rangle-\sqrt{2 / 3}|\uparrow\rangle\left|T_{-}\right\rangle$, where $T_{0}$ and $T$ are two of the triplet states in the right dot. The states $|0\rangle$ and $|1\rangle$ have the nearly same dependence on $\varepsilon$ in the range of detuning at which the qubit is operated (Figures $1 \mathrm{c}$ and $\mathrm{d}$ ), enabling quantum control that is largely insensitive to charge fluctuations. Moreover, electric fields couple to the qubit states and enable high-speed manipulation. ${ }^{16,17,24-27}$ Previously, we experimentally demonstrated non-adiabatic quantum control (direct current (DC)-pulsed gating) of the hybrid qubit, where the manipulation and measurement scheme required the use of a detuning regime that is sensitive to charge noise (with $\varepsilon$ near but not equal to zero —see Figure $1 \mathrm{~d}) .{ }^{18}$ Moreover, DC gating requires abrupt changes in detuning. With a given bandwidth in the transmission line, pulse imperfections arising, e.g., from frequency dependent attenuation, lead to inaccurate control of rotation axes. In this work, we demonstrate resonant microwave-driven control and state-dependent tunnelling readout of the qubit, which together overcome this limitation of DC-pulsed gating and enable full manipulation on the Bloch sphere at a single operating point in detuning that is well-protected from charge noise.

The experiments here are performed in a double dot with a gate design as shown in Figure $1 \mathrm{a}$ and with electron occupations as shown on the stability diagram of Figure $1 \mathrm{~b}$. The electron occupations and energy level alignments used for qubit initialisation, readout and microwave spectroscopy of the qubit states are shown schematically in Figure 1c. All the experiments reported here start with an initial dot occupation of $(1,2)$ and with the system in state $|0\rangle$, prepared at a detuning $\varepsilon \approx 230 \mu \mathrm{eV}$; this detuning is also used for measurement and corresponds to point $M$ in Figure $1 b$. After initialisation, we apply a microwave burst pattern at point $O$, which either coincides with point $M$ or is reached through an adiabatic ramp in detuning (the latter case is illustrated in Figure 1b). The tunnel coupling between the two sides of the double quantum dot mediates an exchange interaction that enables transitions between the qubit states and can be driven by modulating the detuning. ${ }^{16}$ Qubit rotation occurs when the frequency of the applied microwave electric field is resonant with the qubit energy level difference. The measurement point $M$ is chosen so that the Fermi level of the right

\footnotetext{
${ }^{1}$ Department of Physics, University of Wisconsin-Madison, Madison, WI, USA; ${ }^{2}$ Department of Materials Science and Engineering, Yonsei University, Seoul, South Korea and ${ }^{3}$ Department of Materials Science and Engineering, University of Wisconsin-Madison, Madison, WI, USA.

Correspondence: MA Eriksson (maeriksson@wisc.edu)

Received 17 February 2015; revised 13 May 2015; accepted 19 June 2015
} 
a

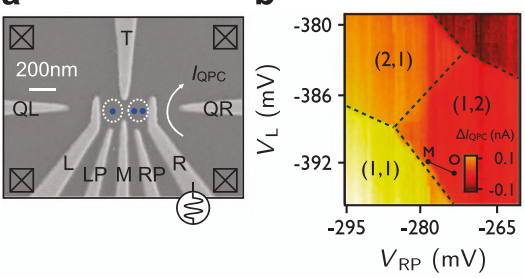

C

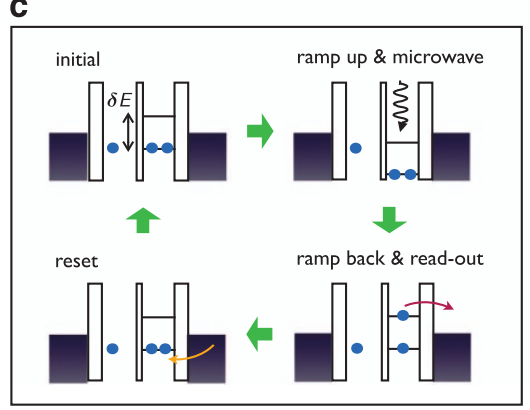

d

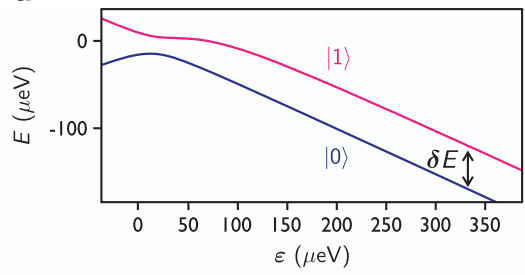

e

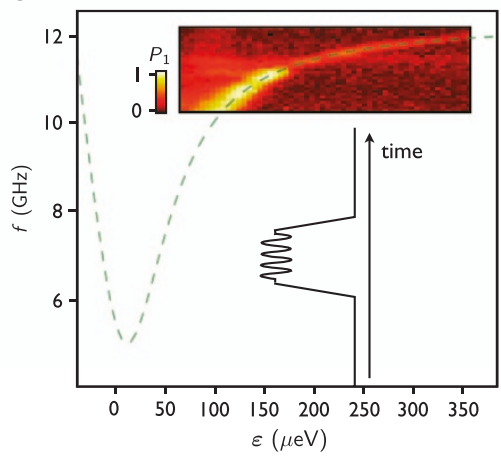

f

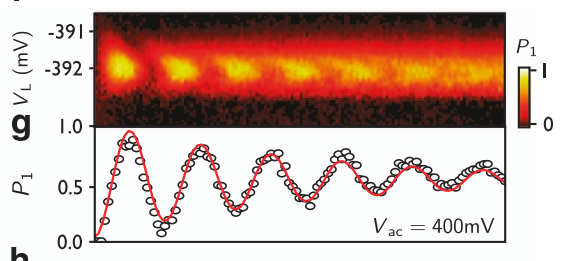

h 1.0
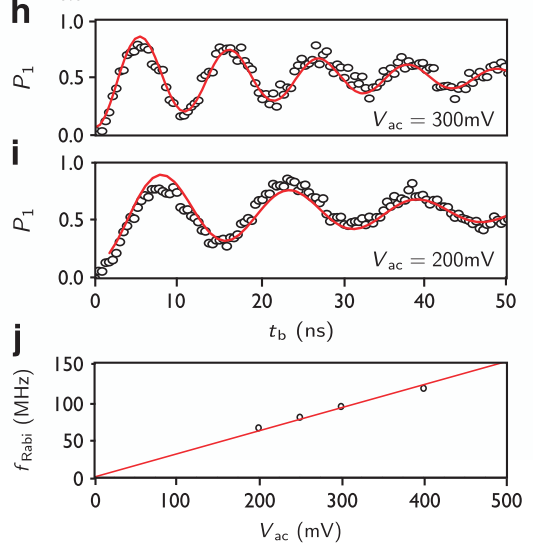

Figure 1. Microwave-driven coherent manipulation and readout of a hybrid qubit in a Si/SiGe double quantum dot device. (a) SEM image and schematic labelling of a device lithographically identical to the one used in the experiment. (b) Charge stability diagram near the $(1,1)-(2,1)-(1,2)$ charge transition, showing the gate voltages used for microwave manipulation $(0)$ and measurement $(\mathrm{M})$. For clarity, a linear background slope was removed from the raw charge-sensing data. (c) Schematic description of the qubit initialisation, manipulation, readout and reset processes. (d) Energy $E$ as a function of detuning $\varepsilon$ for the qubit states, calculated with Hamiltonian parameters measured in ref. 18 (e) Inset: probability $P_{1}$ of the state to be $|1\rangle$ at the end of the driving sequence shown as a function of $\varepsilon$ and the excitation frequency $f$ of the microwaves applied to gate R. In the main panel, the dashed green curve is the energy difference between the ground state and the lowest energy excited state, as determined in ref. 18. (f-j) Coherent Rabi oscillation measurements. (f) $P_{1}$ as a function of the voltage $V_{L}$ and the microwave pulse duration $t_{\mathrm{b}}$ with $f=11.52 \mathrm{GHz}$ and excitation amplitude $V_{\mathrm{ac}}=400 \mathrm{mV}$. (g) Linecut of $P_{1}$ near $V_{\mathrm{L}}=-392 \mathrm{mV}$, showing $\approx 110 \mathrm{MHz}$ Rabi oscillations. The red solid curve is a fit to an exponentially damped sine wave with best fit parameter $T_{\text {Rabi }}=33 \mathrm{~ns}$. (h, i) Rabi oscillation data with microwave amplitude $300 \mathrm{mV}$ (h) and $200 \mathrm{mV}$ (i). (j) Rabi oscillation frequency $f_{\text {Rabi }}$ as a function of $V_{a c}$ with fixed $f=11.52 \mathrm{GHz}$. The good agreement of a linear fit (red line) to the data is strong evidence that the measured oscillations are indeed Rabi oscillations, with the Rabi frequency proportional to the driving amplitude.

reservior is in between the energies of $|0\rangle$ and $|1\rangle$, and we use qubit state-dependent tunnelling to project states $|0\rangle$ and $|1\rangle$ to the $(1,2)$ and $(1,1)$ charge states, respectively. Waiting at point $M$ for $\sim 10 \mu$ s also resets the qubit to state $|0\rangle$, by tunnelling an electron from the reservoir, if needed. Thus, the qubit state population following the microwave burst is measured by monitoring the current $I_{\mathrm{QPC}}(\mathrm{QPC}$, quantum point contact) through the charge-sensing quantum point contact (Figure 1a). Details of the measurement procedure and probability normalisation are in Supplementary Information S1.

\section{MATERIALS AND METHODS}

The details of the Si/SiGe double quantum dot device are presented in refs 28 and 29 . We work in the region of the charge stability diagram where the valence electron occupation of the double dot is $(1,1)$ or $(1,2)$, as confirmed by magnetospectroscopy measurements. ${ }^{29,30}$ All manipulation sequences, including the microwave bursts, are generated by a Tektronix $70002 \mathrm{~A}$ arbitrary waveform generator and are added to the dot-defining DC voltage through a bias tee (Picosecond Pulselabs 5546-107) before being applied to gate $R$. We map the states $|0\rangle$ and $|1\rangle$ to the $(1,2)$ and $(1,1)$ charge occupation states, respectively, leading to conductance changes through the quantum point contact. We measure with a lock-in amplifier (EG\&G model 7265, Oak Ridge, TN, USA) the difference in conductance with and without the applied microwave burst. When converting time averaged conductance differences to the reported probabilities, tunnelling between the $(1,2)$ and $(1,1)$ charge states during the measurement phase is taken into account using the measured times for tunnelling out of $\left(T_{\mathrm{o}} \simeq 200 \mathrm{~ns}\right)$ and into $\left(T_{\mathrm{i}} \simeq 2.1 \mu \mathrm{s}\right)$ the dot. Supplementary Information S1 presents the details of the measurement technique and the probability normalisation.

\section{RESULTS}

We perform microwave spectroscopy of the qubit intrinsic frequency-the energy difference $\delta E$ in Figure 1d-by applying the voltage pulse shown in the inset to Figure 1e. The colour plot in that figure shows the resulting probability of measuring state $|1\rangle$ after applying this pulse to initial state $|0\rangle$. The measured resonance and qubit energy dispersion agrees well with the green dashed curve, which is the calculated energy level diagram with Hamiltonian parameters measured in our previous study. ${ }^{18}$ As is clear from the colour plot in Figure 1e, the linewidth of the resonant peak narrows significantly at $\varepsilon>200 \mu \mathrm{eV}$, becoming much narrower than the resonance in the charge qubit regime $(\varepsilon \approx 0) .{ }^{31}$ This linewidth narrowing corresponds to an increase in the inhomogeneous dephasing time, and it is this range in detuning that corresponds to the hybrid qubit regime. The two states in the right quantum dot that are separated by $\delta E$ most likely correspond to two combinations of the z-valleys, which are weakly mixed by the step in potential at the quantum well interface. ${ }^{32}$

Applying microwave bursts to gate $\mathrm{R}$ in the hybrid qubit regime yields Rabi oscillations, as shown in Figures $1 \mathrm{f}-\mathrm{i}$. The Rabi frequency increases as a function of increasing microwave amplitude $V_{\mathrm{ac}}$ (measured at the arbitrary waveform generator), resulting in Rabi frequencies as high as $100 \mathrm{MHz}$. Figure 1j shows the power dependence of the qubit oscillations, revealing an oscillation frequency that is linear in the applied amplitude, as expected for Rabi oscillations. The speed of the $X$ axis rotation demonstrated here is comparable to electrically manipulated spin rotations in $\mathrm{InSb}$ and InAs, which rely on strong spin-orbit coupling of the host material; ${ }^{33,34}$ here we achieve fast rotations solely through electric field coupling to the qubit states. This 
a

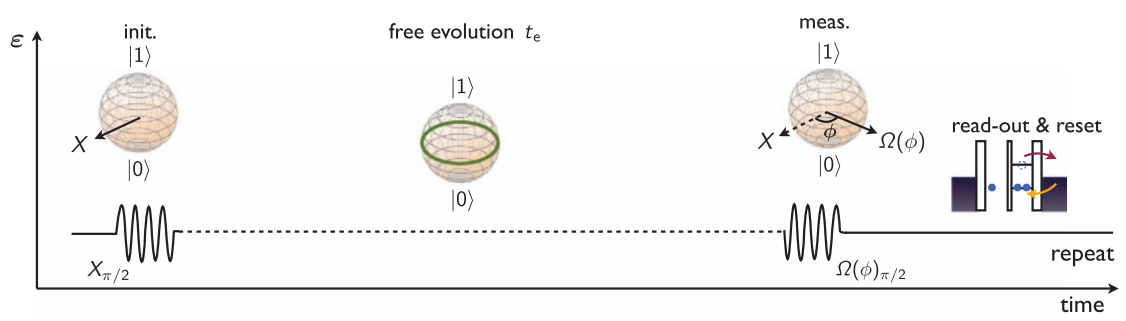

d

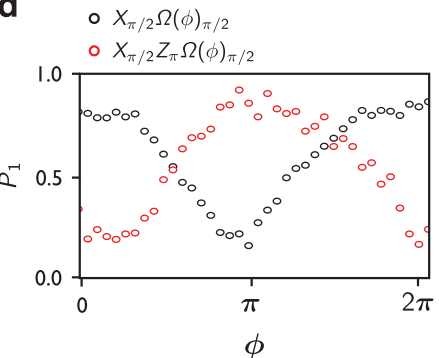

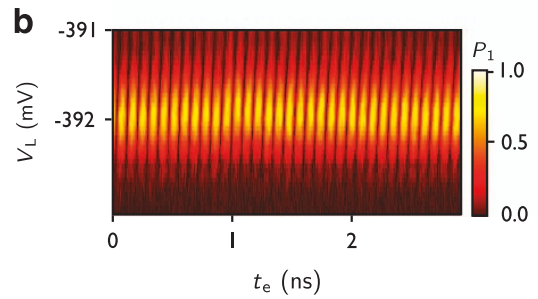

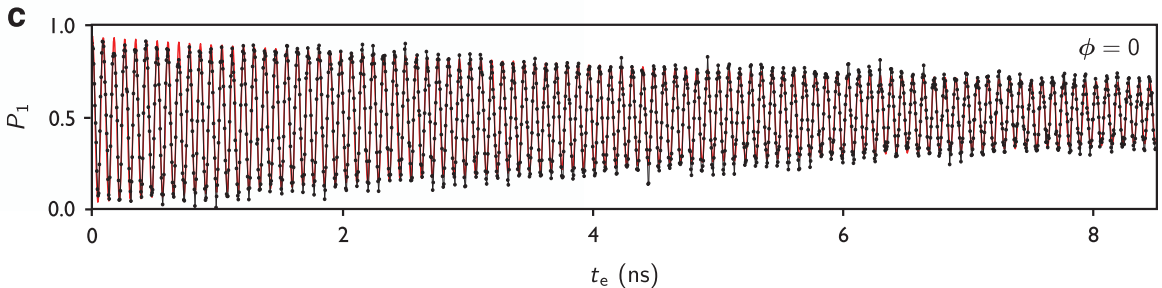

Figure 2. Ramsey fringes and two-axis control of the qubit. (a) Schematic diagram of the pulse sequences used to perform universal control of the qubit. Both the delay $t_{\mathrm{e}}$ and the phase $\phi$ of the second microwave pulse are varied in the experiment. (b, c) Experimental measurement of $Z$ axis rotation. In a Ramsey fringe ( $Z$ axis rotation) measurement, the first $X_{\pi / 2}$ gate rotates the Bloch vector onto the $X-Y$ plane, and the second $X_{\pi / 2}$ gate $(\phi=0)$ is delayed with respect to the first gate by $t_{\mathrm{e}}$, during which time the state evolves freely around the $Z$ axis of the Bloch sphere. (b) $P_{1}$ as a function of $V_{\mathrm{L}}$ and $t_{\mathrm{e}}$ for states initialised near $|Y\rangle$. (c) $P_{1}$ as a function of $t_{\mathrm{e}}$ with fixed $V_{\mathrm{L}}=-391.7 \mathrm{mV}$, showing $\approx 11.52 \mathrm{GHz}$ Ramsey fringes. The red solid curve is a damped sine wave with best fit parameter $T_{2}^{*}=11 \mathrm{~ns}$. (d) Effect of the phase $\phi$ of the second microwave pulse on the state $|Y\rangle$ (by applying $X_{\pi / 2}$ on $|0\rangle$, black), and $|-Y\rangle$ (by applying $X_{\pi / 2}$ and $Z_{\pi}$ on $|0\rangle$, red). The clear oscillation of $P_{1}$ as a function of $\phi$ in both cases demonstrates control over the second rotation axis by control of the phase $\phi$.

a

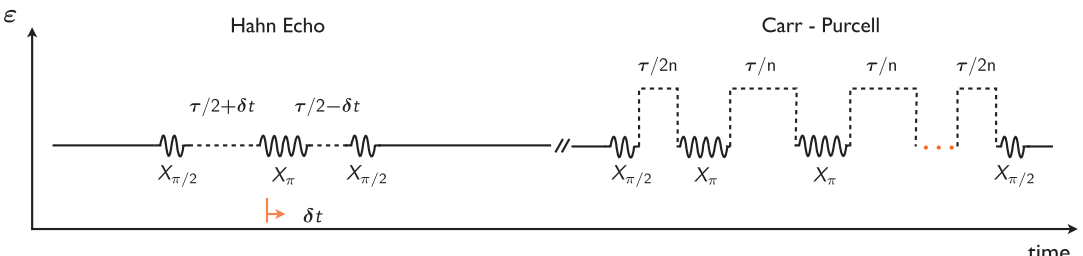

b

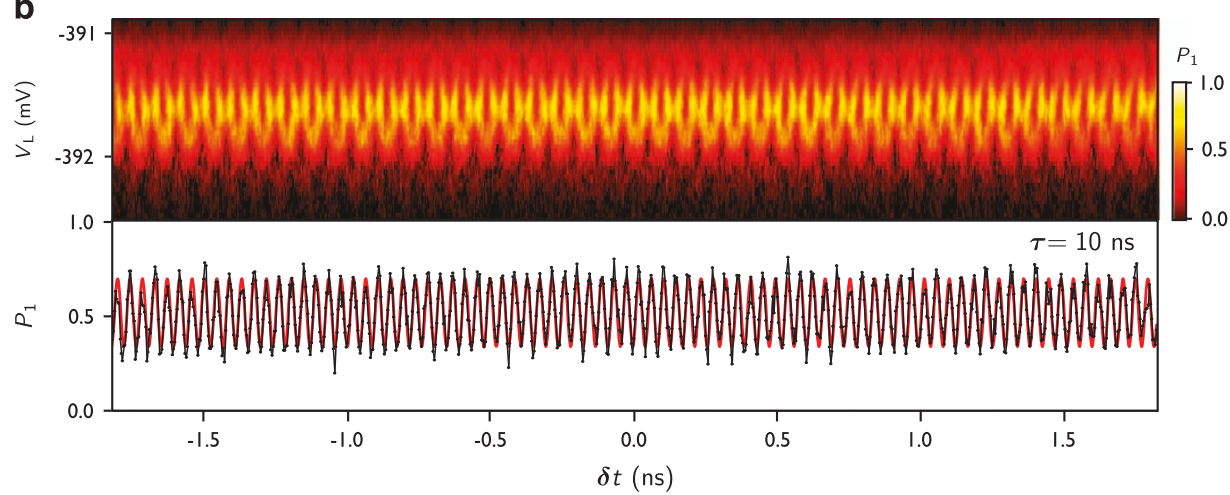

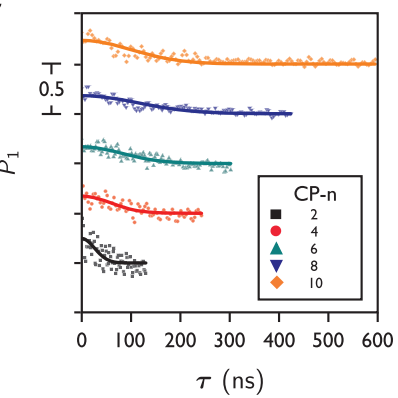

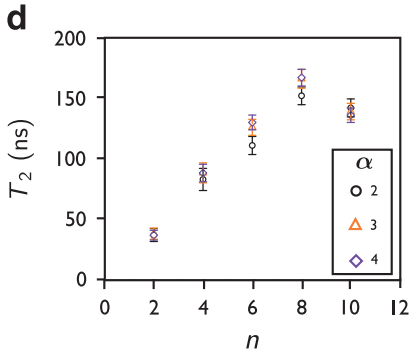

Figure 3. Increasing the coherence time with dynamic decoupling sequences. (a) Schematic pulse sequence for the measurement of Hahn spin echo and CP dynamic decoupling sequences that correct for noise that is static on the timescale of the pulse sequence. ${ }^{19-21}$ Note that for $\mathrm{CP}$ dynamic decoupling, free evolution is performed at $\approx 60 \mu \mathrm{eV}$ more positive detuning than the readout point, in order to prevent tunnelling of the state $|1\rangle$ to the reservoir during the manipulation pulses and free evolution. (b) Typical Hahn echo measurement with fixed total evolution time $\tau=10 \mathrm{~ns}$, showing $P_{1}$ as a function of $V_{L}$ and the delay time $\delta t$ of the $X_{\pi}$ pulse. The bottom panel shows a line cut of $P_{1}$ near $V_{\mathrm{L}}=-391.7 \mathrm{mV}$. The red solid curve shows a fit to a sine wave, and the oscillations of $P_{1}$ as a function of $\delta t$ are at twice the Ramsey frequency $(\approx 23.04 \mathrm{GHz})$; this doubling of frequency is clear evidence of echo, and we measure an echo amplitude of $\approx 0.3$ for $\tau=10 \mathrm{~ns}$ near $\delta t=0$. (c) $P_{1}$ as a function of $\tau$ with fixed $\delta t=0$. The symbols show the data, while the solid curves are the fit to the decay form $P_{1}(\tau)=0.5+A e^{-\left(\tau / T_{2}\right)^{a}}$ with fixed exponent $a=2$ for even numbers $n$ of decoupling $X_{\pi}$ pulses ranging from 2 to 10 . (d) Coherence time $T_{2}$ as a function of $n$ obtained from the fit of CP decay data to the decay form with fixed exponent $a=2$ (black circles), 3 (orange triangles), and 4 (purple diamonds). The resulting $T_{2}$ is insensitive to the choice of $a$ to within the uncertainty. Applying the CP dynamic decoupling sequences increases the coherence time by more than an order of magnitude compared with $T_{2}^{*} \approx 11 \mathrm{~ns}$. 
a
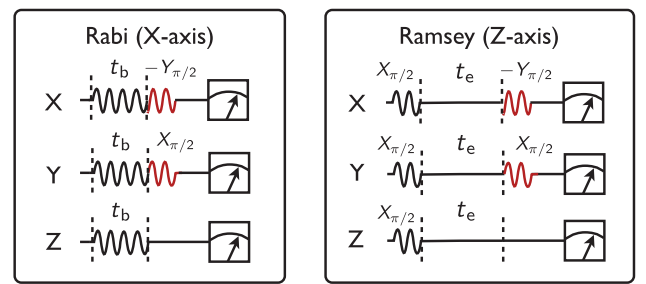

b

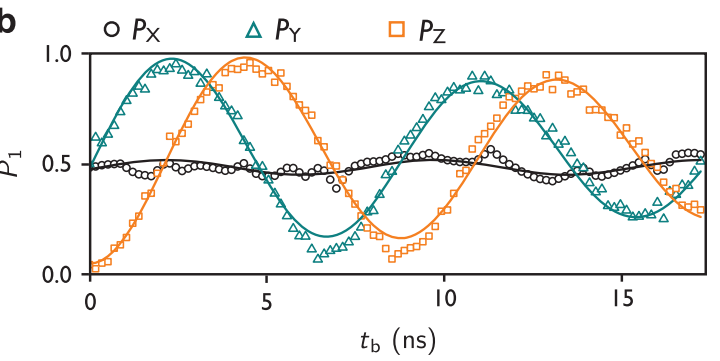

C

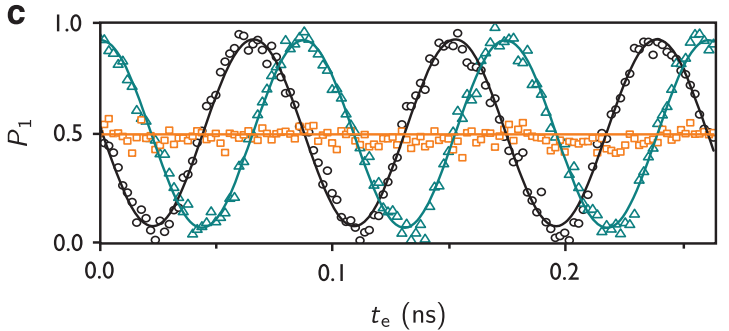

d $X_{\pi / 2}$

bars : Xideal symbols : XML-QPT

$F_{\mathrm{p}}=0.95 \pm 0.01$

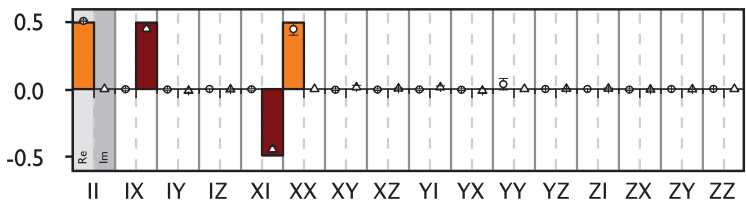

e $X_{\pi}$ $F_{\mathrm{D}}=0.93 \pm 0.01$

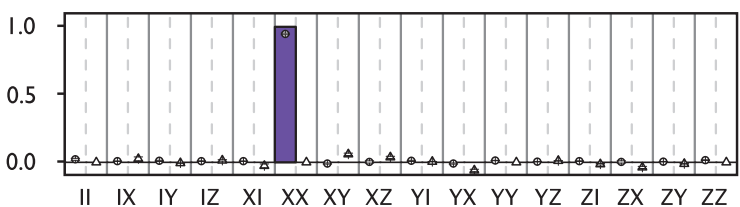

f $Z_{\pi / 2}$ $F_{\mathrm{p}}=0.96 \pm 0.02$

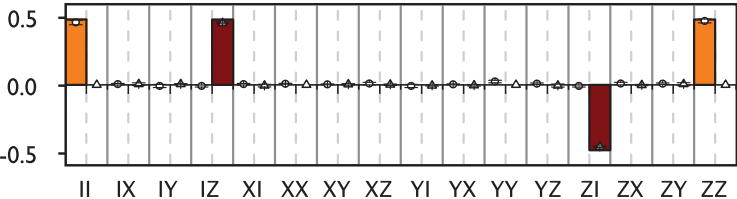

$\operatorname{g~}_{Z_{\pi}}$ $F_{\mathrm{p}}=0.96 \pm 0.02$

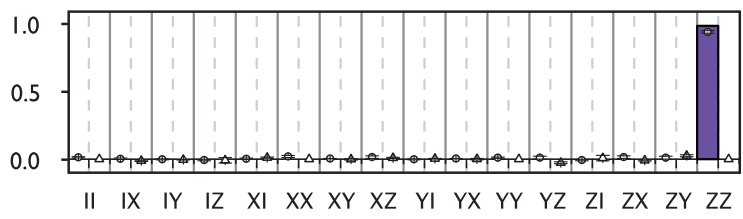

Figure 4. State and quantum process tomography (QPT) of the AC-driven hybrid qubit. (a) Schematic of microwave pulse sequences used for the tomographic characterisation of continuous Bloch vector evolution under $X$ (left panel) and $Z$ (right panel) axis rotations. (b, c) $X$ axis projection $P_{X}$ (green), $Y$ axis projection $P_{Y}$ (orange) and $Z$ axis projection $P_{Z}$ (black) of the Bloch vector evolution under $X$ (b) and $Z$ (c) axis rotations performed in the rotating (lab) frame (see main text for more discussion). (d-g) Real and imaginary parts of the process matrices $X$ (ref. 38) in the Pauli basis $\{1, X, Y, Z\}$ for processes $X_{\pi / 2}(\mathbf{d}), X_{\pi}(\mathbf{e}), Z_{\pi / 2}(\mathbf{f})$ and $Z_{\pi}(\mathbf{g})$ obtained by quantum process tomography with maximum likelihood estimation ${ }^{18,38,39}$ (real: open circles, imaginary: open triangles) compared with corresponding ideal processes (bars). The error bars represent the standard deviation of the result obtained by 10 distinct input and output density matrices chosen from the state tomography data. Process fidelities defined by $F_{\mathrm{p}}=\operatorname{Tr}\left(X_{\text {ideal }} X\right)$ are $93 \%$ for $X_{\pi}$ and $96 \%$ for $Z_{\pi}$ processes, respectively (left panels of e and $\mathbf{g}$ ).

coupling is also highly tunable, since it is determined by the ground and excited state inter-dot tunnel couplings. ${ }^{35}$ Below, we characterize gates with the qubit frequency chosen to be $\approx 11.52 \mathrm{GHz}$.

We characterize the inhomogeneous dephasing time by performing a Ramsey fringe experiment, which also demonstrates $Z$ axis rotations on the qubit Bloch sphere. The microwave pulse sequence is shown schematically in Figure 2a. We first prepare the state $|Y\rangle=\sqrt{1 / 2}(|0\rangle+i|1\rangle)$ by performing an $X_{\pi / 2}$ rotation. $Z$ axis rotation results from the evolution of a relative phase between states $|0\rangle$ and $|1\rangle$, given by $\varphi=-t_{\mathrm{e}} \delta E / \hbar$, where $t_{\mathrm{e}}$ is the time spent between the state preparation and measurement $X_{\pi / 2}$ pulses, the latter of which is used to project the $Y$ axis component onto the $Z$ axis. The final probability $P_{1}$ is measured as described above. Figure $2 \mathrm{~b}$ shows the resulting quantum oscillations as a function of $V_{\mathrm{L}}$, which controls the detuning energy, and $t_{\mathrm{e}}$. In Figure 2b, the frequency of the oscillations increases slightly as $V_{\mathrm{L}}$ becomes more negative, as the qubit energy levels are not quite perfectly independent of detuning. Figure $2 c$ shows a line cut taken near the optimal resonant condition $\left(V_{\mathrm{L}} \approx-392 \mathrm{mV}\right)$, showing clear oscillations in $P_{1}$ consistent with the qubit frequency of $\approx 11.5 \mathrm{GHz}$. By fitting the oscillations to an exponentially damped sine wave (red solid curve), we extract an inhomogeneous dephasing time $T_{2}^{*}=11 \mathrm{~ns}$, consistent with the value measured previously with non-adiabatic pulsed gating on the same device with similar intrinsic qubit frequency. ${ }^{18}$ We estimate the typical tunnelling-out time $T_{\mathrm{o}} \approx 200 \mathrm{~ns}$
(Supplementary Information S1), so that the inhomogeneous coherence time is not likely limited by electron tunnelling to the reservoir during the measurement phase.

Resonant microwave drive also enables arbitrary two-axis control on the $X-Y$ plane of the Bloch sphere by varying the relative phase $\phi$ of the $X_{\pi / 2}$ pulses. Figure $2 d$ shows a measurement of $P_{1}$, demonstrating both two-axis control and phase control. Starting from a maximum (minimum) $P_{1}$ at $\phi=0$, when we apply $X_{\pi / 2} \Omega_{\pi / 2}\left(X_{\pi / 2} Z_{\pi} \Omega_{\pi / 2}\right)$ on the state $|0\rangle, P_{1}$ oscillates as a function of the relative phase $\phi$ that determines the axis of the $\Omega_{\pi / 21 \phi}$ rotation. The deviation from an ideal sinusoidal oscillation stems from limited phase resolution of our method of waveform generation (Supplementary Information S1).

We now turn to echo and dynamic decoupling pulse sequences. Figure 3a shows Hahn echo ${ }^{19-21}$ and CP dynamic decoupling ${ }^{22}$ pulse sequences. Provided that the source of dephasing fluctuates slowly on the timescale of the electron spin dynamics, inserting an $X_{\pi}$ pulse between state initialisation and measurement, which is performed with $X_{\pi / 2}$ gates, corrects for noise that arises during the time evolution. Figure $3 \mathrm{~b}$ shows a typical echo measurement. While keeping the total free evolution time $\tau$ fixed at $10 \mathrm{~ns}$, we sweep the position of the decoupling $X_{\pi}$ pulse to reveal echoed oscillations. ${ }^{21,28}$ In Figure $3 \mathrm{~b}$, the oscillations of $P_{1}$ as a function of $\delta t$ are at twice the Ramsey frequency $\left(2 F_{\text {Ramsey }} \approx 23 \mathrm{GHz}\right)$, as expected for an echo measurement, and the visibility of the oscillations is $\sim 0.35$, because the data were acquired at the relatively long free evolution time of $10 \mathrm{~ns}$. 
Improvement in coherence times can be obtained by implementing CP sequences (Figure 3a), which use multiple $X_{\pi}$ pulses inserted during the free evolution. Since the timescale for the $\mathrm{CP}$ sequence is typically longer than $T_{\mathrm{o}} \approx 200 \mathrm{~ns}$, an adiabatic detuning offset of amplitude $\approx 60 \mu \mathrm{eV}$ was applied to shift point $\mathrm{O}$ (Figure $1 \mathrm{~b}$ ) during free evolution in order to prevent electron tunnelling to the reservoir. In the absence of dephasing, the CP sequence with an even number of $X_{\pi}$ pulses applied on the state $|0\rangle$ yields $P_{1}=1$. The measured $P_{1}$ as a function of $\tau$, shown in Figure 3c, decays exponentially due to dephasing: $P_{1}(\tau)=0.5+A e^{-\left(\tau / T_{2}\right)^{a}}$, where $a$ depends on the frequency spectrum of the dominant noise sources. ${ }^{36}$ Figure $3 \mathrm{~d}$ shows the results of fits as a function of the number $n$ of decoupling $X_{\pi}$ pulses with fixed $a=2,3$ and 4 . The resulting coherence time $T_{2}$ shows more than an order of magnitude improvement (>150 ns) with $n=8$, and the resulting times are approximately independent of the $a$ used in the fit. Beyond $n=8$ we typically observe a decrease in $T_{2}$, which is not completely understood at this time. We expect that optimisation of microwave pulses can increase $T_{2}$ further.

\section{CHARACTERISATION OF THE FIDELITY}

We now present tomographic characterisation of the microwavedriven hybrid qubit. Because this device does not allow singleshot measurement of this qubit, the short number of pulses required for quantum process tomography make that procedure more reliable for the present work than randomized benchmarking, even though at high values of gate fidelity quantum process tomography is typically less reliable than randomised benchmarking. ${ }^{37}$ Here, to reconstruct the time evolution of the single qubit density matrix, we use the microwave pulse sequences shown schematically in Figure 4a to perform repeated state evolution under an $X(Z)$ gate and perform independent $X, Y$ and $Z$ axes projective measurements. For state tomography under $X(Z)$ axis rotation, we prepare initial states near $|0\rangle$ and near $|Y\rangle$. After time evolution under the gate operation, we measure $X, Y$ and $Z$ axes projections of the time-evolved Bloch vector using $-Y(\pi / 2), X(\pi / 2)$, and identity operations, respectively, and measure the resulting $P_{1}$. Note that the pulse sequences shown in Figure $4 a$ represent state tomography in the rotating (laboratory) frame for Rabi (Ramsey) oscillations, because the phase of the second $\pi / 2$ pulses for the Rabi oscillation tomography evolves as the length of Rabi manipulation $t_{\mathrm{b}}$ is increased, whereas the second microwave pulse in the state tomography of the Ramsey fringes has fixed relative phase with respect to the first microwave pulse. Figure $4 \mathrm{~b}$ and $c$ show $X$ (black circles), $Y$ (green triangles) and $Z$ (orange squares) axes projections of the time-evolved Bloch vector under continuous $X(b)$ and $Z(c)$ axes rotation gates.

On the basis of the density matrices obtained from the state tomography, we implement quantum process tomography (QPT) to extract fidelities of single qubit gates on the alternating current (AC)-driven hybrid qubit through the relation, ${ }^{18,38,39}$

$$
\varepsilon(\rho)=\sum_{m, n=1}^{4} \tilde{E}_{m} \rho \tilde{E}_{n}^{\dagger} X_{m n},
$$

where $\varepsilon(\rho)$ is the density matrix specifying the output for a given input density matrix $\rho$, the $\tilde{E}_{m}$ are the basis operators in the space of $2 \times 2$ matrices, and $X$ is the process matrix. Experimentally, four linearly independent input and output states are chosen from continuous evolution of the state under $X$ and $Z$ axes rotations available from the state tomography data set, and the maximum likelihood method ${ }^{18,39}$ is used to determine $X$. Figures $4 \mathrm{~d}-\mathrm{g}$ show the results of QPT (symbols) performed on the $\pi / 2$ and $\pi$ rotations around the $X$ and $Z$ axes and comparison to corresponding ideal rotation process matrices (bars). The error bars of length $\approx 0.01-0.02$ represent the standard deviation of the experimental result obtained by 10 distinct input and output density matrices chosen from the state tomography data. The process matrices $X$ obtained from QPT in the Pauli basis $\left\{l, \sigma_{x}, \sigma_{y}, \sigma_{z}\right\}$ yield process fidelities $F_{\mathrm{p}}=\operatorname{Tr}\left(X_{\text {ideal }} X\right)$ of $93 \%$ and $96 \%$ for $\pi$ rotations around the $X$ and $Z$ axes, respectively. Comparing these results to the process fidelities of $85 \%$ and $94 \%$ for $X$ and $Z$ axis rotations reported previously for the non-adiabatic DC-pulse-gated hybrid qubit, $^{18}$ we find more than a factor of two reduction in the $X$ axis rotation infidelity. The pulses we applied in this work were turned on abruptly, and pulse sequences for consecutive gates were concatenated without gaps, both of which can decrease fidelity; optimisation of the pulse sequences, like that performed in ref. 40, offers opportunities for improvement.

\section{DISCUSSION}

The improvement in overall fidelity of the AC-gated quantum dot hybrid qubit demonstrated here compared with DC-pulsed gating stems mainly from (1) elimination of the need to enter the regime in which the qubit is sensitive to charge noise by using resonant manipulation and tunnelling-based readout, and (2) reduced rotation axis and angle errors because resonant driving with fixed frequency enables more accurate control of these quantities. The AC driving in this work was performed by resonantly modulating the energy detuning between the dots. For this type of modulation, the ratio of manipulation time (Rabi period) to coherence time depends strongly on the strength of ground and excited state tunnel couplings, ${ }^{35}$ and thus we expect that further improvement in fidelity can be achieved by increasing these tunnel couplings, making the energy level dispersion flatter, while maintaining high gate speeds. Moreover, recent theoretical work suggests that dynamically modulating tunnel coupling instead of detuning can enable Rabi frequencies exceeding $1 \mathrm{GHz}$ while keeping long coherence times, enabling achievement of gate fidelites exceeding $99 \% .{ }^{41}$ AC-gating also enables much greater flexibility in the design and operation of quantum gates, as recently demonstrated for quantum control of spins on phosphorous in $\mathrm{Si}_{1}{ }^{42}$ and similar approaches should be possible for both one and two-qubit gates for the hybrid quantum dot qubit.

\section{ACKNOWLEDGEMENTS}

We thank Xuedong Hu, John King Gamble and Brandur Thorgrimsson for useful discussions. This work was supported in part by ARO (W911NF-12-0607) and NSF (PHY-1104660). Development and maintenance of the growth facilities used for fabricating samples is supported by DOE (DE-FG02-03ER46028). This research utilised NSF-supported shared facilities at the University of Wisconsin-Madison.

\section{CONTRIBUTIONS}

DK performed electrical measurements, state and process tomography, and analysed the data with MAE, MF and SNC. DRW developed hardware and software for the measurements. CBS fabricated the quantum dot device. DES and MGL prepared the $\mathrm{Si} / \mathrm{SiGe}$ heterostructure. All authors contributed to the preparation of the manuscript.

\section{COMPETING INTERESTS}

The authors declare no conflict of interest.

\section{REFERENCES}

1 Hanson R, Kouwenhoven LP, Petta JR, Tarucha S, Vandersypen LMK. Spins in few-electron quantum dots. Rev Mod Phys 2007; 79: 1217-1265.

2 Zwanenburg FA, Dzurak AS, Morello A, Simmons MY, Hollenberg LCL, Klimeck G et al. Silicon quantum electronics. Rev Mod Phys 2013; 85: 961.

3 Loss D, DiVincenzo DP. Quantum computation with quantum dots. Phys $\operatorname{Rev} A$ 1998; 57: 120-126.

4 Kane BE. A silicon-based nuclear spin quantum computer. Nature 1998; 393: 133-137. 
5 Elzerman JM, Hanson R, Willems van Beveren LH, Witkamp B, Vandersypen LMK Kouwenhoven LP. Single-shot read-out of an individual electron spin in a quantum dot. Nature 2004; 430: 431-435.

6 Petta JR, Johnson AC, Taylor JM, Laird EA, Yacoby A, Lukin MD et al. Coherent manipulation of coupled electron spins in semiconductor quantum dots. Science 2005; 309: 2180-2184.

7 Koppens FHL, Buizert C, Tielrooij KJ, Vink IT, Nowack KC, Meunier T et al. Driven coherent oscillations of a single electron spin in a quantum dot. Nature 2006; 442 766-771.

8 Foletti S, Bluhm H, Mahalu D, Umansky V, Yacoby A. Universal quantum control of two-electron spin quantum bits using dynamic nuclear polarization. Nat Phys 2009; 5: 903-908.

9 Laird EA, Taylor JM, DiVincenzo DP, Marcus CM, Hanson MP, Gossard AC. Coherent spin manipulation in an exchange-only qubit. Phys Rev B 2010; 82 075403.

10 Gaudreau L, Granger G, Kam A, Aers GC, Studenikin SA, Zawadzki P et al. Coherent control of three-spin states in a triple quantum dot. Nat Phys 2011; 8 54-58.

11 Pla JJ, Tan KY, Dehollain JP, Lim WH, Morton JJL, Jamieson DN et al. A single-atom electron spin qubit in silicon. Nature 2012; 489: 541-545.

12 Medford J, Beil J, Taylor JM, Bartlett SD, Doherty AC, Rashba El et al. Self-consistent measurement and state tomography of an exchange-only spin qubit. Nat Nanotechnol 2013; 8: 654-659.

13 Buch H, Mahapatra S, Rahman R, Morello A, Simmons MY. Spin readout and addressability of phosphorus-donor clusters in silicon. Nat Commun 2013; 4 2017.

14 Maune BM, Borselli MG, Huang B, Ladd TD, Deelman PW, Holabird KS et al. Coherent singlet-triplet oscillations in a silicon-based double quantum dot. Nature 2012; 481: 344-347.

15 Kawakami E, Scarlino P, Ward D, Braakman F, Savage D, Lagally MG et al. Electrical control of a long-lived spin qubit in a Si/SiGe quantum dot. Nat Nanotechnol 2014 9: 666-670.

16 Shi Z, Simmons CB, Prance JR, Gamble JK, Koh TS, Shim Y-P et al. Fast hybrid silicon double-quantum-dot qubit. Phys Rev Lett 2012; 108: 140503.

17 Koh TS, Gamble JK, Friesen M, Eriksson MA, Coppersmith SN. Pulse-gated quantum dot hybrid qubit. Phys Rev Lett 2012; 109: 250503.

18 Kim D, Shi Z, Simmons C, Ward D, Prance J, Koh TS et al. Quantum control and process tomography of a semiconductor quantum dot hybrid qubit. Nature 2014 511: 70-74.

19 Koppens FHL, Nowack KC, Vandersypen LMK. Spin echo of a single electron spin in a quantum dot. Phys Rev Lett 2008; 100: 236802.

20 Vandersypen LMK, Chuang IL. NMR techniques for quantum control and computation. Rev Mod Phys 2005; 76: 1037-1069.

21 Dial OE, Shulman MD, Harvey SP, Bluhm H, Umansky V, Yacoby A. Charge noise spectroscopy using coherent exchange oscillations in a singlet-triplet qubit. Phys Rev Lett 2013; 110: 146804.

22 Bluhm H, Foletti S, Neder I, Rudner M, Mahalu D, Umansky V et al. Dephasing time of GaAs electron-spin qubits coupled to a nuclear bath exceeding $200 \mu \mathrm{s}$. Nat Phys 2011; 7: 109-113.

23 Fowler AG, Mariantoni M, Martinis JM, Cleland AN. Surface codes: towards practical large-scale quantum computation. Phys Rev A 2012; 86: 032324.

24 Ferraro E, Michielis M, Mazzeo G, Fanciulli M, Prati E. Effective hamiltonian for the hybrid double quantum dot qubit. Quantum Inf Process 2014; 13: 1-19.
25 Mehl S. Two-qubit pulse gate for the three-electron double quantum dot qubit Phys Rev B 2015; 91: 035430.

26 Ferraro E, De Michielis M, Fanciulli M, Prati E. Effective Hamiltonian for two interacting double-dot exchange-only qubits and their controlled-not operations. Quantum Inf Process 2015; 14: 47-65.

27 De Michielis M, Ferraro E, Fanciulli M, Prati E. Universal set of quantum gates for double-dot exchange-only spin qubits with intradot coupling. J Phys A: Math Theor 2015; 48: 065304.

28 Shi Z, Simmons CB, Ward DR, Prance JR, Koh TS, Gamble JK et al. Coherent quantum oscillations and echo measurements of a Si charge qubit. Phys Rev $B$ 2013; 88: 075416.

29 Simmons CB, Prance JR, Van Bael BJ, Koh TS, Shi Z, Savage DE et al. Tunable spin loading and $T_{1}$ of a silicon spin qubit measured by single-shot readout. Phys Rev Lett 2011; 106: 156804.

30 Shi Z, Simmons CB, Ward DR, Prance JR, Wu X, Koh TS et al. Fast coherent manipulation of three-electron states in a double quantum dot. Nat Commun 2014; 5: 3020.

31 Kim D, Ward DR, Simmons CB, Gamble JK, Blume-Kohout R, Nielsen E et al. Microwave-driven coherent operations of a semiconductor quantum dot charge qubit. Nat Nanotechnol 2015; 10: 243.

32 Friesen M, Eriksson MA, Coppersmith SN. Magnetic field dependence of valley splitting in realistic Si/SiGe quantum wells. Appl Phys Lett 2006; 89: 202106.

33 Petersson KD, McFaul LW, Schroer MD, Jung M, Taylor JM, Houck AA et al. Circuit quantum electrodynamics with a spin qubit. Nature 2012; 490: 380-383.

34 van den Berg JWG, Nadj-Perge S, Pribiag VS, Plissard SR, Bakkers EPAM, Frolov SM et al. Fast spin-orbit qubit in an indium antimonide nanowire. Phys Rev Lett 2013; 110: 066806.

35 Koh TS, Coppersmith SN, Friesen M. High-fidelity gates in quantum dot spin qubits. Proc Natl Acad Sci USA 2013; 110: 19695-19700.

36 Barthel C, Medford J, Marcus C, Hanson M, Gossard A. Interlaced dynamical decoupling and coherent operation of a singlet-triplet qubit. Phys Rev Lett 2010; 105: 266808

37 Blume-Kohout R. Optimal, reliable estimation of quantum states. New J Phys 2010; 12: 043034.

38 Nielsen MA, Chuang IL. Quantum Computation and Quantum Information. Cambridge University Press: New York, USA, 2000.

39 Chow JM, Gambetta JM, Tornberg L, Koch J, Bishop LS, Houck AA et al. Randomized benchmarking and process tomography for gate errors in a solid-state qubit. Phys Rev Lett 2009; 102: 090502.

40 Kelly J, Barends R, Campbell B, Chen Y, Chen Z, Chiaro B et al. Optimal quantum control using randomized benchmarking. Phys Rev Lett 2014; 112: 240504.

41 Wong $\mathrm{CH}$. Preprint at<http://arXiv.org/pdf/1507.04286>.

42 Laucht A, Muhonen JT, Mohiyaddin FA, Kalra R, Dehollain JP, Freer $\mathrm{S}$ et al. Electrically controlling single-spin qubits in a continuous microwave field. Sci Adv 2015; 1: e1500022.

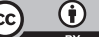

This work is licensed under a Creative Commons Attribution 4.0 International License. The images or other third party material in this article are included in the article's Creative Commons license, unless indicated otherwise in the credit line; if the material is not included under the Creative Commons license, users will need to obtain permission from the license holder to reproduce the material. To view a copy of this license, visit //creativecommons.org/licenses/by/4.0/

Supplementary Information accompanies the paper on the npj Quantum Information website (http://www.nature.com/npjqi) 